\begin{tabular}{|c|l|}
\hline Title & Coupled-mode stop bands of acoustic phonons in semiconductor superlattices \\
\hline Author(s) & Tamura, S.; Wolfe, J. P. \\
\hline Citation & $\begin{array}{l}\text { Physical Review B, 35(5), 2528-2531 } \\
\text { https://doi.org/10.1103/PhysRevB.35.2528 }\end{array}$ \\
\hline Issue Date & 1987-02-15 \\
\hline Doc URL & http://hdl.handle.net/2115/5959 \\
\hline Rights & Copyright $\odot 1987$ A merican Physical Society \\
\hline Type & article \\
\hline File Information & PRB35-5.pdf \\
\hline
\end{tabular}

Instructions for use 


\title{
Coupled-mode stop bands of acoustic phonons in semiconductor superlattices
}

\author{
S. Tamura* and J. P. Wolfe \\ Department of Physics and Materials Research Laboratory, University of Illinois at Urbana-Champaign, \\ Urbana, Illinois 61801 \\ (Received 12 November 1986)
}

\begin{abstract}
Dispersion relations of acoustic phonons in the (001) GaAs/AlAs superlattice are calculated for arbitrary propagation directions using the continuum approximation with the elastic anisotropy included. We predict the existence of forbidden band gaps inside the folded Brillouin zone in addition to those at the zone boundary and the zone center. They occur by the mixing of different phonon modes having propagation directions oblique to the interfaces. The phonon transmission rates are calculated for superlattices with a finite number of periods, and significant dips are predicted for frequencies corresponding to zone-center, zone-boundary, and coupled-mode stop bands.
\end{abstract}

Much work has been devoted to the understanding of the acoustic properties of superlattices. ${ }^{1-9}$ The periodic structure in a superlattice along the growth direction is known to produce Brillouin-zone (BZ) folding and the appearance of gaps in the phonon spectrum, i.e., phonon stop bands, for wave vectors satisfying the Bragg condition. Phonons with frequencies in these band gaps are attenuated in the superlattice. The presence of the phonon stop bands has so far only been studied for phonon propagation perpendicular to the interfaces of the superlattice. The first experimental observations of Bragg scattering of high-frequency acoustic phonons were made by Narayanamurti et al. ${ }^{2}$ several years ago. They used GaAs/ $\mathrm{Al}_{x} \mathrm{Ga}_{1-x} \mathrm{As}$ superlattices with (001) and (111) surfaces and found dips in the phonon transmission at frequencies corresponding to the forbidden gaps at the zone boundaries. More recently, Koblinger et al. ${ }^{7}$ have observed similar effects for amorphous $\mathrm{SiO}_{2} / \mathrm{Si}$ superlattices, also for propagation perpendicular to the interfaces. An interesting prospect is to vary the propagation direction of the incident phonons with respect to the superlattice planes, analogous to scanning the angle of an optical diffraction grating. Anisotropies associated with phonon focusing and mode conversion at an interface make this a challenging problem.

In this Rapid Communication we examine the phonon dispersion relation for oblique propagation and predict a new type of stop band which occurs inside the folded zone. These new forbidden gaps are due to "anticrossings" of phonon modes with mixed polarizations. The mixing of the bulk-mode polarizations occurs by the presence of the elastic anisotropy combined with reflection and transmission at an interface. The predominant polarizations of the transmitted and reflected phonons in, say, GaAs and $\mathrm{Al}_{x} \mathrm{Ga}_{1-x} \mathrm{As}$ layers are not necessarily the same. Energy gaps occur when a folded branch approaches another branch with some of the same polarization character. In addition to predicting the phonon dispersion relations at oblique angles, we have calculated the transmission rates in the superlattice with a finite number of periods. It displays prominent dips at frequencies corresponding to these interior-zone band gaps.

Consider the superlattice configuration depicted in Fig. 1(a). Perfectly flat interfaces parallel to the $x_{\|}$plane are assumed. The plane-wave solution for the displacement field $U$ of the phonons in each layer $(l=1$ or 2$)$ has the following form:

$$
\mathbf{U}(l)=A \exp \left[i\left(\mathbf{k}_{\|} \cdot \mathbf{x}_{\|}+k_{\|} \kappa_{3} z-\omega t\right)\right],
$$

where $\mathbf{k}_{\|}=k_{\|}\left(\kappa_{1}, \kappa_{2}\right)$ is the two-dimensional wave vector parallel to the interface (i.e., $k_{\|}=\left|\mathbf{k}_{\|}\right|, \kappa_{1}^{2}+\kappa_{2}^{2}=1$ ), e is the unit polarization vector, $A$ is the amplitude, and $\omega$ is the angular frequency. In Eq. (1), $\kappa_{3}$ determines the propagation characteristics of the phonons perpendicular to the interfaces and is related to the angle $\theta$ of the wave vector measured from the $z$ axis by $\kappa_{3}=\cot \theta$.

For any given values of $\kappa_{1}$ and $\kappa_{2}$ the polarization vector e and $\kappa_{3}$ in each layer are obtained by solving the

(a)

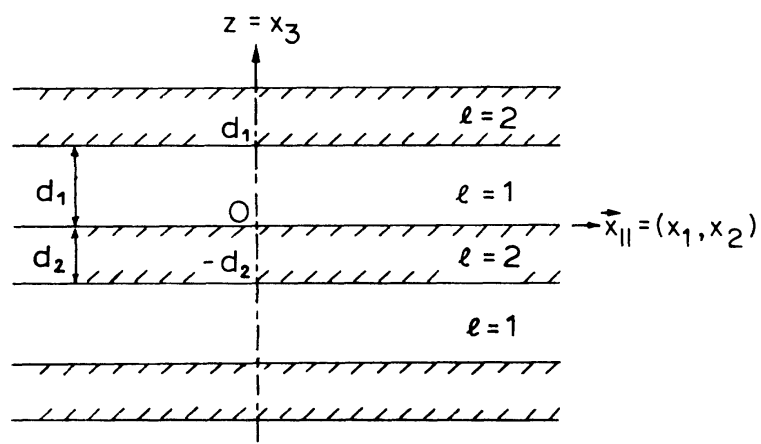

(b)

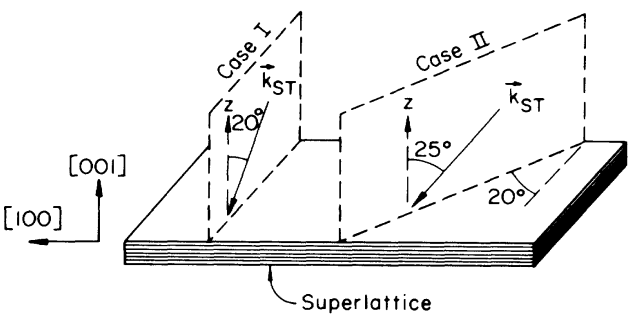

FIG. 1. (a) Schematic of a superlattice configuration consisting of alternating layers with thickness $d_{1}$ and $d_{2}$. (b) Saggital planes and angles of incidence (or transmission) of ST-phonon wave vectors in GaAs layer assumed for the calculations of dispersion relations. See text. 
Christoffel equation

$$
\left[C_{i j m n}(l)_{\kappa_{j} \kappa_{n}}-\rho(l) v^{2} \delta_{i m}\right] e_{m}=0, i=1,2,3,
$$

where $C_{i j m n}$ is the elastic-constant tensor, $\rho$ is the mass density of the medium, and $v$ is the velocity given by $v=\omega / k_{\|}$. (A sum over repeated indices is assumed.) Since the secular equation derived from Eq. (2) is of degree 6 in $\kappa_{3}$, there are six solutions for $\kappa_{3}$, three of which correspond to the transmitted $(+z$ propagating) waves and another three correspond to the reflected $(-z$ propagating) waves of different polarizations. Hence, the actual displacement field $\mathbf{U}$ in each layer is the linear combination of six terms of the form of Eq. (1) with different value of $\kappa_{3}$. Here, we note that $\mathbf{k}_{\|}$and $v$ are the same for all twelve reflected and transmitted waves at a single interface and $v$ is related to the phase velocity $c$ by Snell's law, $v=c / \sin \theta$. The displacement field $\mathbf{U}$ and stress field, defined by

$$
S_{3 m}(l)=C_{3 m j n}(l) \partial_{j} U_{n}(l), m=1,2,3,
$$

must satisfy the appropriate continuity and periodicity conditions at an interface $z=z_{0}{ }^{10}$ They are given by

$$
\begin{aligned}
& W_{m}\left(l=1 ; z_{0}\right)=W_{m}\left(l=2 ; z_{0}\right), m=1,2,3, \\
& W_{m}\left(l ; z_{0}+D\right)=e^{i q D} W_{m}\left(l ; z_{0}\right), m=1,2,3,
\end{aligned}
$$

where $W_{m}$ represents both $U_{m}$ and $S_{3 m}, D=d_{1}+d_{2}$ is the superlattice period, and $q$ is the superlattice wave number describing the propagation perpendicular to the interfaces.

Now, Eqs. (4) and (5) give twelve linear equations to be solved for twelve amplitudes- six amplitudes $A$ in each layer, $l=1$ and $l=2$. In order that all of these amplitudes are not identically zero, the determinant of the $12 \times 12$ matrix consisting of the coefficients of $A$ 's must vanish. This gives the eigenvalue equation for $x=e^{i q D}$. More explicitly, $x$ is given by the eigenvalue of the $6 \times 6$ matrix $M$ defined by

$$
\begin{aligned}
M= & {\left[H\left(l=2 ; z=-d_{2}\right)\right]^{-1} H\left(l=1 ; z=d_{1}\right) } \\
& \times[H(l=1 ; z=0)]^{-1} H(l=2 ; z=0) .
\end{aligned}
$$

The elements of the $6 \times 6 \mathrm{H}$ matrices are components of the displacement and stress fields. Explicitly, we have

$$
H(l)=\left(\begin{array}{c}
\left(u_{m}^{(J)}\right),\left(\tilde{u}_{m}^{(J)}\right) \\
\left(s_{m}^{(J)}\right),\left(\tilde{s}_{m}^{(J)}\right)
\end{array}\right), m, J=1,2,3,
$$

where $u_{m}^{(J)}$ and $s_{m}^{(J)}\left(\tilde{u}_{m}^{(J)}\right.$ and $\left.\tilde{s}_{m}^{(J)}\right)$ are the $+z$ propagating ( $-z$ propagating) components of the displacement and stress fields with unit amplitudes, and $J$ is the mode index. For each mode, $\kappa_{3}$ is different. Note that these fields are functions of the wave number $k_{\|}$, thus $H$ and $M$ are functions of the phonon frequency $v$.

As a typical example, we show in Fig. 2 the dispersion curves $v(q)$ for phonons with a given propagation direction in the (100) plane (saggital plane) of a (001) $\mathrm{GaAs} / \mathrm{AlAs}$ superlattice. The widths of the layers are assumed to be $40 \AA$ (i.e., $d_{1}=d_{2}=40 \AA$ ). In case I, a $20^{\circ}$ angle of incidence (or transmission) is chosen for the slow-transverse (ST) -phonon wave vector in a GaAs layer

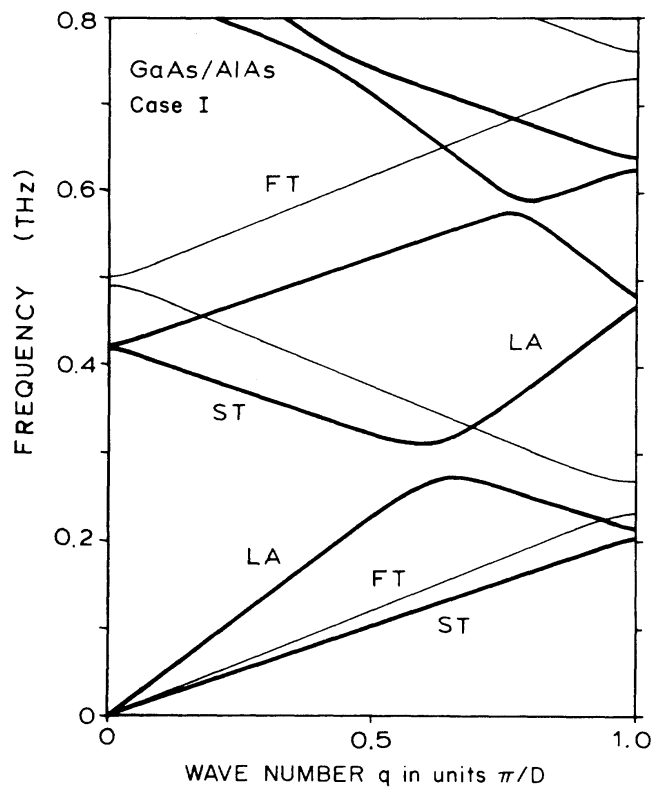

FIG. 2. Dispersion curves of acoustic phonons propagating in the (001) GaAs/AlAs superlattice with layer thickness of $d_{1}=d_{2}=40 \AA$. [Case I of Fig. 1(b).] The wave number $q_{\max }$ of the zone boundary phonons is $3.927 \times 10^{6} \mathrm{~cm}^{-1}$.

[see Fig. 1(b)]. Using Snell's law, fast transverse (FT) and longitudinal acoustic (LA) phonons with the same $k_{\|}$ and $\omega$ have angles of incidence $22.8^{\circ}$ and $37.2^{\circ}$, respectively. ${ }^{11}$ For these propagation directions, FT phonons are polarized perpendicular to the saggital plane and, hence, they are decoupled from the LA and ST phonons. The ST and LA phonons, however, are coupled to each other. At low frequencies the dispersion curves of the three phonon modes are well separated. This means that the transmitted and reflected waves in each layer are almost identical to the transverse or longitudinal waves in the bulk, and the mixing of the different modes is very small. As the frequency increases the dispersion curve of the ST phonons reaches the zone boundary and is folded back into the zone, producing a forbidden gap at the boundary. This folded ST dispersion curve encounters the dispersion curve of the LA branch. Because of the oblique propagation, both of these modes contain some longitudinal and transverse character (i.e., they are not pure shear or compression waves) and the mixing of the polarizations increases with increasing frequency. Consequently, the dispersion curves of the ST and LA modes repel each other and form a new band gap. More specifically, in this region of the $q-v$ plane the longitudinal (transverse) lattice vibrations incident to the interface produce mainly transverse (longitudinal) reflections. Because of this mixing of the modes the polarizations of the lattice vibrations in each layer cannot be identified as predominantly longitudinal or transverse. For frequencies in the band gap the transmitted and reflected waves in each layer carry exactly the same magnitude of energy flux perpendicular to the interfaces, and therefore there is no net energy transmission to adjacent layers.

If the saggital plane of the phonons is not oriented along 
a symmetry direction, all three modes of the phonons couple due to the elastic anisotropy and much more complicated mixing of the branches is obtained. Figure 3 shows typical dispersion curves in such a case. Here we are still considering a GaAs/AlAs superlattice with (001) crystal surface. The saggital plane of the wave vector is rotated $20^{\circ}$ away from the (100) plane, as indicated by case II in Fig. 1(b). The angle of incidence for the ST wave vector is chosen to be $25^{\circ}$ in GaAs, which by Snell's law gives $29.2^{\circ}$ and $52.0^{\circ}$, respectively, for FT and LA incident angles in the same layer. In this case, mixing of the FT branch with the ST and LA branches occurs in addition to the mixing of the latter two branches. A number of coupled-mode forbidden bands are generated (e.g., regions 1, 3, and 5 for the FT mode). It should be noted that in the longwavelength approximation these dispersion curves apply to any thickness $D=d_{1}+d_{2}$ with $d_{1}=d_{2}$; only the scale of $v$ is changed.

The dispersion curves of Figs. 2 and 3 were obtained for an ideal superlattice with an infinite number of periods. We now address the question of whether the coupled-mode stop bands can lead to observable effects in superlattices with a finite number of periods. Typically, a superlattice is grown on a thick substrate (usually GaAs). One can imagine an experiment where phonons transmitted through the substrate are incident on the superlattice and are detected on the opposite side of the superlattice. We define the transmission rate as the ratio of the acoustic Poynting vector perpendicular to the interface to that of the incident phonons from the substrate. For a multilayered system such as a superlattice, the transmission rate can be calculated by applying the transfer-matrix technique to elastic waves in anisotropic media. In the present calculations, the number of periods is assumed to be twen-

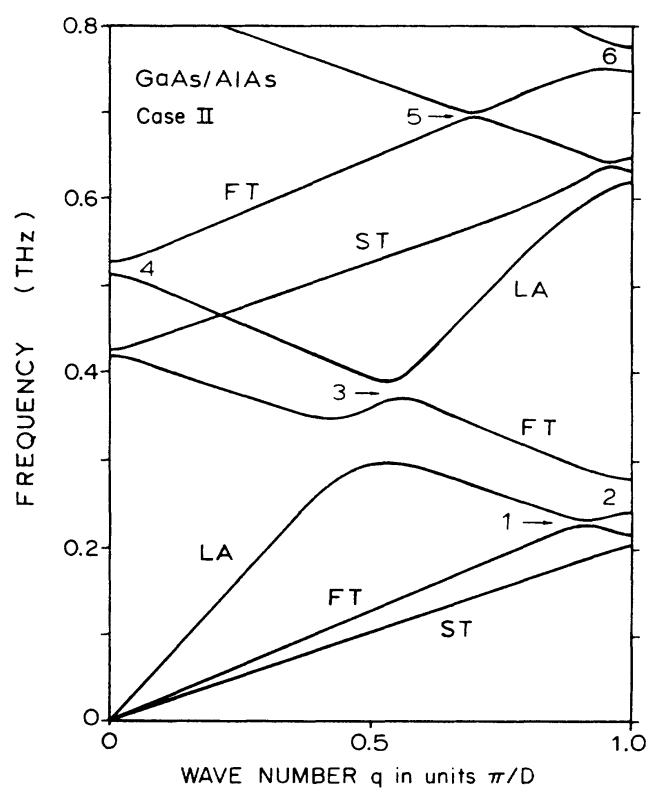

FIG. 3. Dispersion curves of acoustic phonons propagating in the (001) GaAs/AlAs superlattice with $d_{1}=d_{2}=40 \AA$. [Case II of Fig. 1(b).] $q_{\max }=3.927 \times 10^{6} \mathrm{~cm}^{-1}$. The numbers labeled 1 to 6 indicate the stop bands of FT phonons. ty and the thickness of individual GaAs and AlAs layers is $40 \AA$ each. The directions of the wave vectors are the same as those for which the dispersion curves of Fig. 3 are obtained.

The calculated frequency dependence of the transmission rate is given in Fig. 4 for the case of FT phonons. Effects of the phonon stop bands are clearly evident even in a twenty-period superlattice. The frequencies at which the stop bands in Fig. 3 occur agree very well with the dips in transmission. The dips in the transmission rate indicated by 2,4 , and 6 correspond to zone-boundary and zonecenter reflections, and those labeled 1,3 , and 5 are due to coupled-mode stop bands.

As the angles of the transmission of the waves in the softer layer ( $\mathrm{GaAs}$ ) increase past a certain critical angle, the transmitted waves in the harder layer (AlAs) become evanescent waves which are localized near the interfaces (i.e., the corresponding $\kappa_{3}$ 's are complex). These "pseudosurface waves" do not carry energy perpendicular to the interfaces. The study of such waves has been made for single interfaces. ${ }^{12,13}$ Remarkably, in the case of superlattices, we still find dispersion curves similar to those in Figs. 2 and 3. This is one of the interesting results of our calculations, namely, that continuous phonon bands are formed even when the phonons in one layer become evanescent. This can be best illustrated for the simple case in which the FT phonons propagate in the (100) saggital plane with polarization vector parallel to the [010] direction. For these propagation directions the dispersion relation takes the following analytic form. By defining $\kappa_{3}(l=1 ; J$ $=\mathrm{FT})=\kappa$ and $\kappa_{3}(l=2 ; J=\mathrm{FT})=\kappa^{\prime}$ we have

$$
\begin{aligned}
\cos (q D)= & \cos \left(k_{\|} \kappa d_{1}\right) \cos \left(k_{\|} \kappa^{\prime} d_{2}\right) \\
& -\frac{1+\sigma^{2}}{2 \sigma} \sin \left(k_{\| \kappa d_{1}}\right) \sin \left(k_{\|} \kappa^{\prime} d_{2}\right),
\end{aligned}
$$

where $k_{\|}=\omega / v$ and

$$
\sigma=\rho(2) \kappa^{\prime}\left(1+\kappa^{2}\right) /\left[\rho(1) \kappa\left(1+\kappa^{2}\right)\right] .
$$

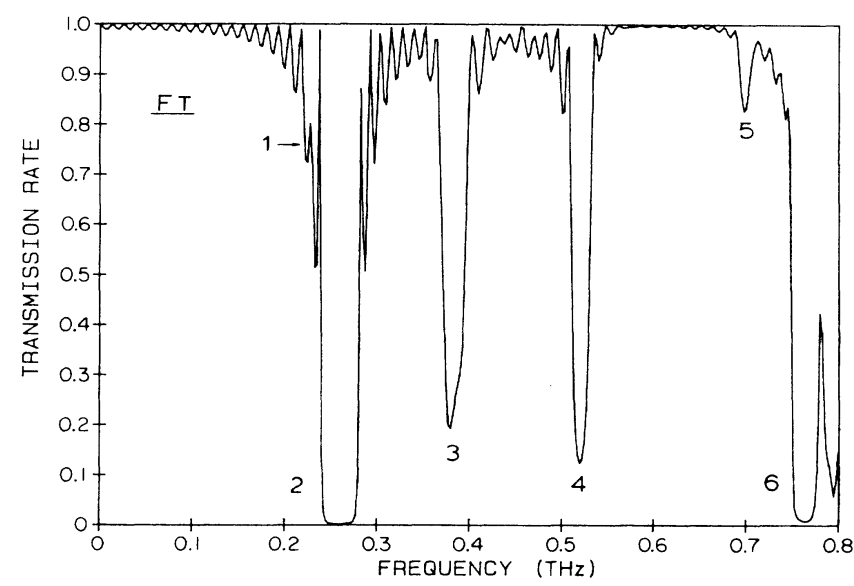

FIG. 4. The transmission rate of the FT phonons in the (001) $\mathrm{GaAs} / \mathrm{AlAs}$ superlattice with the number of periods equal to 20 and $d_{1}=d_{2}=40 \AA$. The propagation direction is the same as that for Fig. 3 (case II). The dips labeled 1 to 6 correspond to the band gaps in Fig. 3. 
In the (001) GaAS/AlAs superlattice the FT phonons in the AlAs layer become evanescent waves for the angle of the transmission larger than $58^{\circ}$ in the GaAs layer. In this case $\kappa$ is real but $\kappa^{\prime}$ is purely imaginary. However, Eq. (8) still holds if we note that the sinusoidal functions of the imaginary arguments are hyperbolic functions of the real arguments. Thus the right-hand side of Eq. (8) is real valued even in this case, and its modulus takes a value smaller than unity for a certain finite interval of the transmission angles in the GaAs layer.

To conclude, we have shown that for oblique propagation band gaps in the phonon spectrum are generated inside the folded $\mathrm{BZ}$ in addition to those at zone boundary and zone center. The interior-zone stop bands are due to the mixing of the different phonon modes. It was also shown that the phonon transmission rate through a super- lattice with a finite number of periods has prominent dips at frequencies corresponding to these band gaps. In the present work we have employed the long-wavelength approximation to predict those results. At frequencies for which the discreteness of the lattice becomes important we should further include the effects of the dispersion in the bulk materials. Actually dispersive propagation is much more complicated but undoubtedly coupled-mode band gaps are still present in this high frequency regime.

One of us (S.T.) acknowledges a travel grant by the Department of Education, Science and Culture of Japan. This work was supported by the U.S. National Science Foundation under the Materials Research Laboratory Grant No. NSF-DMR-83-16981.
*Permanent address: Department of Engineering Science, Hokkaido University, Sapporo 060, Japan.

${ }^{1}$ A. S. Baker, Jr., J. L. Merz, and A. C. Gossard, Phys. Rev. B 17, 3181 (1978).

${ }^{2}$ V. Narayanamurti, H. L. Stormer, M. A. Chin, A. C. Gossard, and W. Wiegmann, Phys. Rev. Lett. 43, 2012 (1979).

${ }^{3}$ C. Colvard, R. Merlin, M. V. Klein, and A. C. Gossard, Phys. Rev. Lett. 45, 298 (1980).

${ }^{4}$ S. K. Yip and Y. C. Chang, Phys. Rev. B 30, 7037 (1984).

${ }^{5}$ C. Colvard, T. A. Gant, M. V. Klein, R. Merlin, R. Fischer, H. Morkoc, and A. C. Gossard, Phys. Rev. B 31, 2080 (1985).

${ }^{6}$ M. J. Kelly, J. Phys. C 18, 5963 (1985).

${ }^{7}$ O. Koblinger, J. Mebert, E. Dittrich, S. Döttinger, and W. Eisenmenger, in Proceedings of the Fifth International Conference of Phonon Scattering in Condensed Matter, edited by A. C. Anderson and J. P. Wolfe (Springer, New York,
1986), p. 156.

${ }^{8}$ R. E. Camley, B. Djafari-Rouhani, L. Dobrzynski, and A. A. Maradudin, Phys. Rev. B 27, 7318 (1983).

${ }^{9}$ M. Babiker, D. R. Tilly, E. L. Albuquerque, and C. E. T. Goncalves daSilva, J. Phys. C 18, 1269 (1985).

${ }^{10}$ S. M. Rytov, Zh. Eksp. Teor. Fiz. 29, 605 (1955) [Sov. Phys. JETP 2, 466 (1956)].

${ }^{11} \mathrm{We}$ have used $\rho=5.36 \mathrm{~g} / \mathrm{cm}^{3}, C_{11}=11.88 \times 10^{11} \mathrm{dyn} / \mathrm{cm}^{2}$, $C_{12}=5.38 \times 10^{11} \mathrm{dyn} / \mathrm{cm}^{2}, C_{44}=5.94 \times 10^{11} \mathrm{dyn} / \mathrm{cm}^{2}$ for $\mathrm{GaAs}$ and $\rho=3.76 \mathrm{~g} / \mathrm{cm}^{3}, C_{11}=12.02 \times 10^{11} \mathrm{dyn} / \mathrm{cm}^{2}, C_{12}=5.70$ $\times 10^{11} \mathrm{dyn} / \mathrm{cm}^{2}, C_{44}=5.89 \times 10^{11} \mathrm{dyn} / \mathrm{cm}^{2}$ for AlAs. See S. Adachi, J. Appl. Phys. 58, R3 (1985).

${ }^{12}$ A. G. Every, G. L. Koos, and J. P. Wolfe, Phys. Rev. B 29, 2190 (1984).

${ }^{13}$ A. G. Every, Phys. Rev. B 33, 2719 (1986). 